\title{
Current Therapeutic Concepts in Hepatorenal Syndrome
}

\section{Gurpratap Sandhu' ${ }^{*}$, Nancy Gupta ${ }^{2}$ and Harkirat Singh ${ }^{3}$}

${ }^{1}$ University of Pittsburgh Medical Center, Division of General Internal Medicine, Pittsburgh, USA

${ }^{2}$ Department of Internal Medicine, Westchester Medical Center, Valhalla, New York, USA

${ }^{3}$ University of Pittsburgh Medical Center, Division of Gastroenterology and Hepatology, Pittsburgh, USA

Keywords: Hepatorenal syndrome; Cirrhosis; Survival; Terlipressin; Management

Hepatorenal syndrome (HRS) is a complication associated with advanced cirrhosis. In patients with cirrhosis and ascites, HRS comprises of about $20 \%$ of the cases of acute renal failure, with pre-renal $(37 \%)$, acute tubular necrosis $(42 \%)$ and post-renal $(0.3 \%)$ comprising of the remaining cases $[1,2]$. Hence, it remains a diagnosis of exclusion. There are two types of HRS that are identified: Type 1: Acute deterioration in renal function, with rise in serum creatinine to a value twice the baseline and $>2.5 \mathrm{mg} / \mathrm{dl}$ within 2 weeks. Precipitating factors include bacterial infections (57\%), gastrointestinal hemorrhage (36\%) and large volume paracentesis without adequate plasma expansion (7\%) [3]. Type 2: Slow and gradual deterioration of kidney function, with serum creatinine $>1.5 \mathrm{mg} / \mathrm{dl}$, often associated with refractory ascites. It is usually not associated with precipitating factors and represents functional renal failure associated with cirrhosis. Type 2 has higher mortality because of its rapidly progressive course. It results in the death of the patients who do not die of other complications of cirrhosis. The pathophysiologic differences between the two types are not well understood and continue to be an area of intense research. There are no specific tests to differentiate HRS from other causes of renal failure in cirrhosis and the diagnosis is that of exclusion. Criteria for diagnosis of HRS have been proposed and include: Chronic or acute hepatic disease with advanced hepatic failure and portal hypertension; serum creatinine $>1.5 \mathrm{mg} / \mathrm{dl}$; absence of any other apparent cause for the acute kidney injury like shock, nephrotoxic drugs or post obstructive renal failure; lack of improvement in renal function after volume expansion with intravenous albumin ( $1 \mathrm{~g} / \mathrm{kg}$ of body weight per day up to $100 \mathrm{~g} /$ day) for at least two days and withdrawal of diuretics; and absence of parenchymal disease.

The ideal treatment for HRS is the treatment of underlying cause of cirrhosis. Improvement in liver function from abstinence of alcohol, antiviral treatment for hepatitis B or liver transplantation can lead to dramatic improvement in renal function. However, if improvement in liver function in not expected in the short term, medical therapy should be instituted to possibly recover some kidney function. The currently therapeutic approach to HRS includes the administration of vasoconstrictive agents with albumin, transjugular intrahepatic portosystemic shunt (TIPS) and dialysis.

\section{Vasoconstrictors}

The currently available vasoconstrictors available for treatment of HRS include vasopressin analogues and alpha adrenergic agents like noradrenaline, midodrine and octreotide. Vasoconstrictors improve central hypovolemia through splanchnic vasoconstriction, leading to decreased systemic levels of vasopressor hormones like renin, resulting in decreased renal vascular resistance and improving renal perfusion in patients with HRS. In a recent meta-analysis [4,5], a significant reversal of HRS was observed in the terlipressin group vs placebo patients in HRS patients; the pooled OR of HRS reversal rate was 8.09 (95\% CI, $3.521 ; 18.59 ; \mathrm{p}=0.001)$. However, the reduction in mortality is seen with type I, but not type II HRS. Terlipressin is given as an intravenous bolus ( 1 to $2 \mathrm{mg}$ every four to six hours), and albumin is given for two days as an intravenous bolus $(1 \mathrm{~g} / \mathrm{kg}$ per day [100 $\mathrm{g}$ maximum]), followed by 25 to 50 grams per day until terlipressin therapy is discontinued. When terlipressin is not available, combination of midodrine, octreotide, and albumin can be used. Some recent studies have shown that noradrenaline may be equally effective in improving renal function when compared to terlipressin [6-8]. Noradrenaline is significantly cheaper than terlipressin, but is limited in its use to the intensive care setting. Norepinephrine is given intravenously as a continuous infusion ( 0.5 to $3 \mathrm{mg} / \mathrm{hr}$ ) with the goal of raising the mean arterial pressure by $10 \mathrm{mmHg}$, and albumin is given for at least two days as an intravenous bolus ( $1 \mathrm{~g} / \mathrm{kg}$ per day [100 g maximum]).

\section{Transjugular Intrahepatic Portosystemic Shunt (TIPS)}

An alternative approach to medical therapy for HRS is TIPS and has been primarily used in patients with refractory ascites. TIPS involves placing a side-to-side portocaval shunt that links the portal and hepatic veins, reducing the portal pressure. It results in some of the blood that is pooled in the splanchnic circulation being returned to the systemic circulation, thus improving the hemodynamics. There have been few studies assessing the role of TIPS in HRS. In a study by Brensing et al. [9], they treated 31 HRS patients, $77 \%$ of the patients exhibited sustained improvement in glomerular filtration rate. Testino et al treated 9 patients with severe acute alcoholic hepatitis with TIPS and found that serum creatinine improved to $1.6 \pm 0.6$ compared to 5.2 \pm 0.9 at baseline [10]. Unfortunately, many patients with HRS are too sick to undergo TIPS. TIPS is associated with complications including an increase in the rate of hepatic encephalopathy, worsening of liver function, shunt stenosis and bleeding. Given the risks associated with the procedure, TIPS should be considered a last resort in patients with HRS, not responding to vasoconstrictors.

\section{Dialysis}

Dialysis can be used as bridge to transplant or when there is a possibility of improvement in liver function. In addition, dialysis improves the priority score on the wait list for renal transplantation in United States. However, initiation of dialysis can be of limited benefit in patients with HRS type 1 who are not candidates for liver transplant. This is in part due to the poor life expectancy, high morbidity and mortality associated with the initiation of dialysis, in part due to increased risk of hemorrhage and hypotension.

\section{Conclusion}

HRS remains frequent among patients with end stage liver disease

${ }^{*}$ Corresponding author: Gurpratap Sandhu, Division of General Internal Medicine, University of Pittsburgh Medical Center, Division of General Internal Medicine, Pittsburgh, USA, E-mail: sandhugs@upmc.edu

Received: May 28, 2014; Accepted: May 30, 2015; Published: June 05, 2015

Citation: Sandhu G, Gupta N, Singh H (2015) Current Therapeutic Concepts in Hepatorenal Syndrome. J Nephrol Ther 5: e112. doi:10.4172/2161-0959.1000e112

Copyright: $\odot 2015$ Sandhu $\mathrm{G}$,et al. This is an open-access article distributed under the terms of the Creative Commons Attribution License, which permits unrestricted use, distribution, and reproduction in any medium, provided the original author and source are credited. 
Citation: Sandhu G, Gupta N, Singh H (2015) Current Therapeutic Concepts in Hepatorenal Syndrome. J Nephrol Ther 5: e112. doi:10.4172/2161$0959.1000 \mathrm{e} 112$

and without therapy, most patients with HRS have extremely poor prognosis. Most patients die within weeks of onset of renal impairment. The outcome of patients HRS, as well as recovery of kidney function, is strongly dependent upon reversal of the hepatic failure. Convincing evidence has been gathered over the years showing combined therapy with vasoconstrictors such as terlipressin and albumin improves renal function and survival in patients with HRS. Dialysis and TIPS can be offered to patients with HRS who are refractory to therapy with vasoconstrictors.

\section{References}

1. Angeli P, Morando F, Cavallin M, Piano S (2011) Hepatorenal syndrome. Contributions to nephrology 174: 46-55.

2. Gines P, Guevara M, Arroyo V, Rodes J (2003) Hepatorenal syndrome. Lance 362: 1819-1827.

3. Pham PT, Pham PC, Rastogi A, Wilkinson AH (2005) Review article: current management of renal dysfunction in the cirrhotic patient. Aliment Pharmacol Ther 21: 949-961.
4. Fabrizi F, Dixit V, Messa P, Martin P (2009) Terlipressin for hepatorenal syndrome: A meta-analysis of randomized trials. Int J Artif Organs 32: 133-140.

5. Fabrizi F, Dixit V, Martin P (2006) Meta-analysis: terlipressin therapy for the hepatorenal syndrome. Aliment Pharmacol Ther 24: 935-944.

6. Duvoux C, Zanditenas D, Hézode C, Chauvat A, Monin JL, et al. (2002) Effects of noradrenalin and albumin in patients with type I hepatorenal syndrome: a pilot study. Hepatology 36: 374-380.

7. Ghosh S, Choudhary NS, Sharma AK, Singh B, Kumar P, et al. (2013) Noradrenaline vs terlipressin in the treatment of type 2 hepatorenal syndrome: a randomized pilot study. Liver Int 33: 1187-1193.

8. Singh V, Ghosh S, Singh B, Kumar P, Sharma N, et al. (2012) Noradrenaline vs. terlipressin in the treatment of hepatorenal syndrome: a randomized study. Hepatology 56: 1293-1298.

9. Brensing KA, Textor J, Perz J, Schiedermaier P, Raab P, et al. (2000) Long term outcome after transjugular intrahepatic portosystemic stent-shunt in nontransplant cirrhotics with hepatorenal syndrome: a phase II study. Gut 47: 288295.

10. Testino G, Leone S, Ferro C, Borro P (2012) Severe acute alcoholic hepatitis and hepatorenal syndrome: role of transjugular intrahepatic portosystemic stent shunt. J Med Life 5: 203-205. 